\title{
A Finite Element Modeling of the Impact of Internal Erosion on the Stability of a Dike
}

\author{
Jie Yang ${ }^{1}$; Zhen-Yu Yin ${ }^{2}$; Pierre-Yves Hicher ${ }^{3}$; and Farid Laouafa ${ }^{4}$
}

${ }^{1}$ LUNAM Univ., Ecole Centrale de Nantes, GeM UMR CNRS 6183, Nantes, France; INERIS, Verneuil en Halatte, France. E-mail: jie.yang@ec-nantes.fr

${ }^{2}$ LUNAM Univ., Ecole Centrale de Nantes, GeM UMR CNRS 6183, Nantes, France. E-mail: zhenyu.yin@ec-nantes.fr

${ }^{3}$ LUNAM Univ., Ecole Centrale de Nantes, GeM UMR CNRS 6183, Nantes, France. E-mail: pierre-yves.hicher@ec-nantes.fr

${ }^{4}$ INERIS, Verneuil en Halatte, France. E-mail: farid.laouafa@ineris.fr

\begin{abstract}
This paper presents a numerical model of internal erosion based on the approach of continuous porous media. The soil skeleton saturated by a pore fluid is treated as the superposition of four constituents in interaction: solid skeleton, erodible fines, fluidized particles, and fluid. The detachment and transport of fine particles are modeled by the mass exchange between solid and fluid phases. In order to take into account the influence of the change of void ratio induced by internal erosion on the soil skeleton, a critical state based constitutive model is used to calculate the effective stress-strain response of the soil skeleton. This coupled hydro-mechanical analysis is applied to study the impact of erosion on a dike. The numerical simulations show the progressive development of internal erosion within the foundation of the dyke. The effect of the mechanical degradation due to internal erosion is demonstrated by evaluating the factor of safety of the dyke slope by means of the shear strength reduction method.
\end{abstract}

\section{INTRODUCTION}

Internal erosion is a phenomenon of progressive degradation of soil induced by hydrodynamic forces, reducing progressively its mechanical properties, and eventually leading to failure. This phenomenon has been studied by laboratory testing and numerical modeling (Reddi et al. 2000; Sterpi 2003; Cividini et al. 2004; Wan et al. 2004; Cividini et al. 2009; Fujisawa et al. 2010). However, most of the numerical studies of coupled hydro-mechanical analysis for internal erosion have used a linear elastic constitutive model, whereas a more realistic elastoplastic constitutive model should be considered in order to discuss the stability of earth structures during internal erosion. 
This paper presents a coupled hydro-mechanical analysis for internal erosion using the approach of continuous porous media (Vardoulakis et al. 1996; Stavropoulou et al. 1998). The soil skeleton saturated by a pore fluid is treated as the superposition of four constituents in interaction. The internal erosion of the soil skeleton and the transport of fine particles by pore water are modeled by the mass exchange between solid and fluid phases. The stress-strain relationship for the solid skeleton is calculated by a critical state based constitutive model (Yin et al. 2013) which can take into account the progressive degradation of the soil mechanical properties induced by internal erosion. The balance equations of the material system are implemented into a finite element code. The discretized equations are linearized and iteratively solved by the Newton-Raphson method to ensure the equilibrium at the current configuration. The finite element code is then applied for evaluating the influence of internal erosion on a dike slope stability by calculating the safety factor by using the shear strength reduction method.

\section{DEFINITION OF THE HYDRO-MECHANICAL EQUATIONS}

\section{Mass balance and particle transport}

The mass balance equations are based on the porous media theory (Boer 2000; Schrefler 2002). The saturated porous medium is modeled as a material system consisting of the stable fabric of the solid skeleton $\left(s_{s}\right)$, erodible fines $\left(f_{s}\right)$, fluidized particles $\left(f_{c}\right)$ and fluid $\left(f_{f}\right)$. In current conditions, fines can either behave as a fluid-like (described as fluidized particles $f_{c}$ ) or a solidlike (described as erodible fines $f_{s}$ ) material. Thus a liquid-solid phase transition process is taken into account in the present model by a mass/volume production term in the corresponding partial mass/volume balances of $f_{s}$ and $f_{c}$.

In a given Representative Elementary Volume (REV) with the volume $\mathrm{d} V$ consisting of the four constituents, the volume fraction of a single constituent $\alpha$ is expressed as:

$$
n^{\alpha}=\frac{d V^{\alpha}}{d V}
$$

with $\alpha=\left\{s_{s}, f_{s}, f_{c}, f_{f}\right\}$.

The local form of the partial balance of mass for the $\alpha$ phase is given as (Schaufler et al.2013):

$$
\partial_{t}\left(\rho^{\alpha}\right)+\operatorname{div}\left(\rho^{\alpha} \mathbf{v}^{\alpha}\right)=\hat{\rho}^{\alpha}
$$

Notice that in this study the hydro-mechanical dispersion is disregarded. The partial density $\rho^{\alpha}$ is defined as the ratio between the mass $d m^{\alpha}$ of the phase $\alpha$ with respect to the total volume $d V$ of the REV, which leads to a relation between partial densities $\rho^{\alpha}$ and the effective densities $\rho^{\alpha R}$ :

$$
\rho^{\alpha}=\frac{d m^{\alpha}}{d V}=\frac{d m^{\alpha}}{d V^{\alpha}} \frac{d V^{\alpha}}{d V}=\rho^{\alpha R} n^{\alpha}
$$


The constraint for the mixture mass production $\sum_{\alpha} \hat{\rho}^{\alpha}=0$ in the system guarantees that there is no mass production in the total mixture of the assembly. The partial mass balance is then reduced to a partial volume balance:

$$
\partial_{t}\left(n^{\alpha}\right)+\operatorname{div}\left(n^{\alpha} \mathbf{v}^{\alpha}\right)=\hat{n}^{\alpha}
$$

where $\hat{n}^{\alpha}$ is the volumetric mass exchange term which will be discussed in the following part.

Moreover, it is assumed that fluid and fluidized particles have at any time the same velocity. The solid skeleton is assumed to be deformable and non-erodible. The porosity field $\phi$, the amount of erodible fines $f_{s}$, the concentration of the fluidized particles $f_{c}$ are defined as follows:

$$
\begin{gathered}
\phi=\frac{d V_{v}}{d V}=\frac{d V^{f_{f}}+d V^{f_{c}}}{d V}=n^{f_{f}}+n^{f_{c}} \\
f_{s}=\frac{n^{f_{s}}}{n^{s_{s}}+n^{f_{s}}} \\
f_{c}=\frac{n^{f_{c}}}{\phi}
\end{gathered}
$$

Considering the transformation of the state of fines from part of the solid skeleton to fluidized particles $\hat{n}^{f_{c}}=-\hat{n}^{f_{s}}, \hat{n}^{f}=0$, the mass balance equations are given by the following expressions:

$$
\begin{gathered}
\partial_{t} \phi=-\hat{n}^{f_{s}} \\
\partial_{t}(c \phi)+\operatorname{div}(c \mathbf{q})=\hat{n}^{f_{c}} \\
\operatorname{div}(\mathbf{q})+\partial_{t}\left(\varepsilon_{v}\right)=0
\end{gathered}
$$

\section{Constitutive equation for mass exchange}

Equations (6)-(8) are not sufficient for solving a boundary value problem since a constitutive relation for the mass exchange term is needed. A constitutive law for the rate of eroded mass, suggesting that erosion is driven by the discharge of the fluidized particles $f_{c}|\mathbf{q}|$, is given by the following relation (Vardoulakis et al. 1996; Uzuoka et al. 2012):

$$
\hat{n}^{f_{s}}=\lambda_{e} f_{c}|\mathbf{q}|
$$

where the coefficient $\lambda_{e}$ has the inverse length dimension and must be determined experimentally.

\section{Darcy's law}

In this study, the flow in the porous medium is considered to be governed by Darcy's law which states that the flow rate is driven by the gradient of the pore fluid pressure:

$$
\mathbf{q}=-\frac{k}{\eta_{k} \bar{\rho}} \operatorname{grad}(p)
$$

where $\eta_{k}$ is the kinematic viscosity of the fluid, $p$ is the pore fluid pressure and $\bar{\rho}$ is the partial density of the mixture defined as:

$$
\bar{\rho}=f_{c} \rho_{s}+\left(1-f_{c}\right) \rho_{f}
$$


with $\rho_{s}$ the density of solid and $\rho_{f}$ the density of fluid. The permeability $k$ of the porous medium depends on the porosity $\phi$. In this study, the permeability $k$ is defined as (Stavropoulou et al. 1998):

$$
k=k_{0} \frac{\phi^{k_{1}}}{(1-\phi)^{k_{2}}}
$$

\section{Constitutive equations for the solid phase}

The mechanical behavior of the solid skeleton is reproduced by a critical state based constitutive model (Yin et al. 2013) in order to take into account the hydro-mechanical coupling effect induced by internal erosion.

The total strain rate is decomposed into elastic and plastic parts:

$$
\dot{\varepsilon}_{i j}=\dot{\varepsilon}_{i j}^{e}+\dot{\varepsilon}_{i j}^{p}
$$

The elastic behavior is assumed to be isotropic with the bulk modulus $K$ having the form proposed by Richart et al. (1970):

$$
\begin{gathered}
\dot{\varepsilon}_{i j}^{e}=\frac{1+v}{3 K(1-2 v)} \sigma_{i j}^{\prime}-\frac{v}{3 K(1-2 v)} \sigma_{k k}^{\prime} \delta_{i j} \\
K=K_{0} \cdot p_{a t} \frac{(2.97-\mathrm{e})^{2}}{1+e}\left(\frac{p^{\prime}}{p_{a t}}\right)^{\zeta}
\end{gathered}
$$

where $K_{0}$ and $\zeta$ are elastic parameters; $v$ is Poisson's ratio; $p^{\prime}$ is the mean effective stress; $p_{a t}$ is the atmospheric pressure used as reference pressure $\left(p_{a t}=101.325 \mathrm{kPa}\right)$.

The plastic strain is based on the flow rule:

$$
\dot{\varepsilon}_{i j}^{p}=d \lambda \frac{\partial g}{\partial \sigma_{i j}^{\prime}}
$$

with the yield surface for shear sliding expressed as:

$$
f=\frac{q}{p^{\prime}}-\frac{M_{p} \varepsilon_{d}^{p}}{k_{p}+\varepsilon_{d}^{p}}
$$

where $f$ is the yield function; $q$ is the deviatoric stress; $k_{p}$ relates to the plastic shear modulus; $M_{p}$ is the stress ratio corresponding to the mobilized peak strength determined by the mobilized peak friction angle $\phi_{p}\left(M_{p}=6 \sin \left(\phi_{p}\right) /\left(3-\sin \left(\phi_{p}\right)\right)\right.$ in compression $) ; \varepsilon_{d}^{p}$ is the deviatoric plastic strain. The potential surface including the stress-dilatancy expression is written as follows:

$$
\begin{aligned}
& \frac{\partial g}{\partial \sigma_{i j}^{\prime}}=\frac{\partial g}{\partial p^{\prime}} \frac{\partial p^{\prime}}{\partial \sigma_{i j}^{\prime}}+\frac{\partial g}{\partial s_{i j}} \frac{\partial s_{i j}}{\partial \sigma_{i j}^{\prime}} \text { with } \\
& \frac{\partial g}{\partial p^{\prime}}=D\left(M_{p t}-\frac{q}{p^{\prime}}\right) ; \frac{\partial g}{\partial s_{i j}}=\sqrt{\frac{3}{2}} \frac{s_{i j}}{\sqrt{s_{i j} s_{i j}}}
\end{aligned}
$$

where $s_{i j}=\sigma_{i j}^{\prime}-p^{\prime} \delta_{i j} ; D$ is the stress-dilatancy parameter; $M_{p t}=6 \sin \left(\phi_{p t}\right) /\left(3-\sin \left(\phi_{p t}\right)\right)$ can be calculated from the mobilized phase transformation friction angle $\phi_{p t}$. 
The mobilized peak friction angle and the phase transformation friction angle are linked to the critical friction angle $\phi_{\mu}$ (corresponding to the critical state line, CSL, in the $p^{\prime}-q$ plane), and the critical void ratio $e_{c}$ (obtained from the critical state line, CSL, in the $e-\log p^{\prime}$ plane) as follows:

$$
\begin{gathered}
\tan \phi_{p}=\frac{e_{c}}{e} \tan \phi_{\mu} ; \tan \phi_{p t}=\left(\frac{e_{c}}{e}\right)^{-1} \tan \phi_{\mu} \\
e_{c}=e_{r e f}-\lambda \ln \frac{p^{\prime}}{p_{r e f}}
\end{gathered}
$$

where $e_{r e f}$ is the reference critical void ratio corresponding to $p_{r e f}=p_{a t} ; \lambda$ is the slope of the CSL in the $e-\log p^{\prime}$ plane.

The plastic multiplier, $d \lambda$, can be calculated in a conventional way according to plasticity theory:

$$
d \lambda=\frac{\left[\frac{\partial f}{\partial \sigma_{i j}}\right]^{T}}{\left[\frac{\partial f}{\partial \sigma_{i j}}\right]^{T}\left[D_{i j k l} \frac{\partial g}{\partial \sigma_{k l}}\right]-\frac{\partial f}{\partial \varepsilon_{d}^{p}} \frac{\partial g}{\partial q}}
$$

Combining Equations (13) to (21), the stress-strain relationship can be obtained and is expressed in terms of effective stresses applied on the solid skeleton.

\section{Hydromechanical coupling effect}

Conceptually, the hydro-mechanical coupling effect can be divided into two parts. In the first part, the internal erosion results in an evolution of the material porosity, which leads to the change of permeability controlled by Eq.(12). As a consequence, the pore pressure and the effective stresses will be redistributed. In the second part, the loss of fine particles due to seepage flow affects the mechanical behavior of the soil.

\section{SIMULATION OF THE IMPACT OF INTERNAL EROSION ON A DIKE}

To illustrate the suggested model, the stability of a dike slope was analyzed. In this example, a karst collapse underneath the foundation led to a cavity through which the underground water could flow out. Erosion takes place due to local hydraulic gradients in the vicinity of the cavity. The computations are aimed at evaluating the impact of internal erosion on the dike slope stability. The geometry and dimension of this numerical example are schematically shown in Figure 1 according to the typical cross section of the dike of Val d'Orléans (Alboresha 2016). The water level is at the top of the alluvium which is fully saturated whereas the dike is considered dry. The problem is analyzed under the assumption of plane strain condition. The material parameters are summarized in Table 1. 


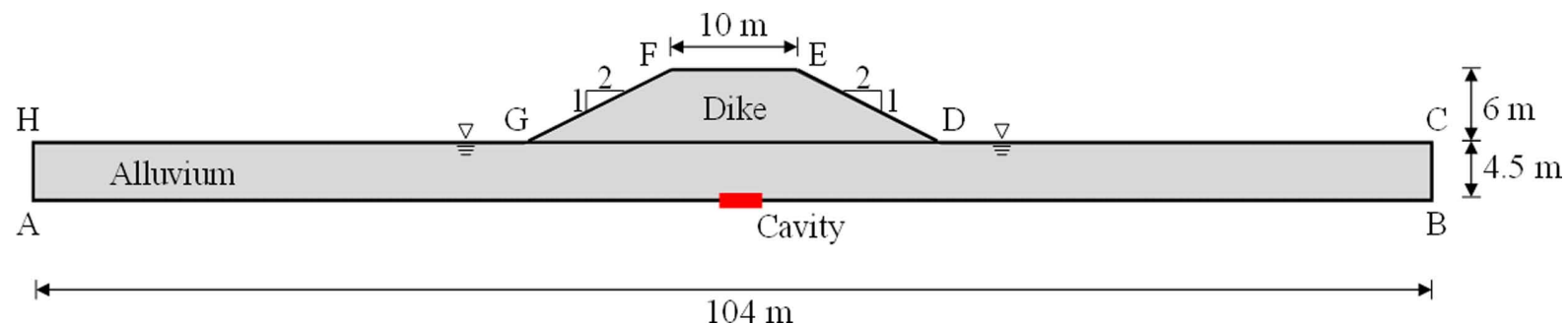

Figure 1. Schematic configuration of the dike.

Table 1. Material parameters

\begin{tabular}{lcllll}
\hline \multicolumn{2}{l}{ Physical and mechanical properties } & \multicolumn{3}{l}{ Hydraulic properties } & \\
\hline Density of fluid & $\rho_{f}$ & $1.0 \mathrm{~g} / \mathrm{cm}^{3}$ & Kinematic viscosity of fluid & $\eta_{k}$ & $5.0 \mathrm{E}-6 \mathrm{~m}^{2} \mathrm{~s}^{-1}$ \\
Density of solids & $\rho_{s}$ & 2.65 & Intrinsic permeability & $k_{0}$ & $5 \mathrm{E}-9 \mathrm{~m}^{2}$ \\
Bulk modulus & $K_{0}$ & $130 p_{\text {at }}$ & Erosion coefficient & $\lambda_{e}$ & $1 \mathrm{E}-3 \mathrm{~m}^{-1}$ \\
Shear modulus & $G_{0}$ & $78 p_{\text {at }}$ & Initial concentration & $f_{c, 0}$ & 0.01 \\
Critical void ratio of $p_{r e f}$ & $e_{r e f}$ & 0.936 & Initial erodible fines in soil skeleton & $f_{s, 0}$ & 0.25 \\
CSL parameter & $\lambda$ & 0.0936 & Initial void ratio & $\phi_{0}$ & 0.3939 \\
CSL parameter & $\kappa$ & 0.365 & Permeability parameter & $k_{l}$ & 3 \\
Critical friction angle & $\phi_{u}$ & $31.6^{\circ}$ & Permeability parameter & $k_{2}$ & 2 \\
\hline
\end{tabular}

Two representative results are presented in Figures 2 and 3. Figure 2 shows the evolution of the porosity. As internal erosion takes place, the porosity which is initially uniform (see Figure 3a) increases with time (see Figure 3b-3c). The increase is particularly critical in the vicinity of the cavity, which leads to a highly eroded zone. As the dike is dry, the erosion process takes place only in the alluvium.

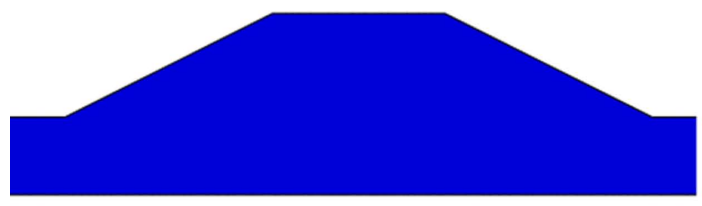

(a) Initial

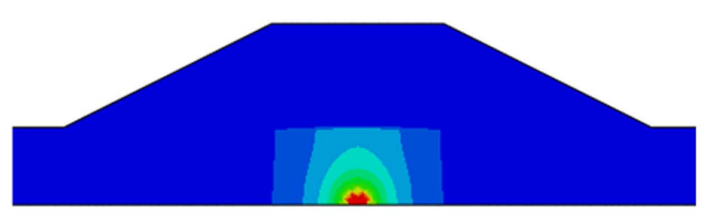

(c) $2.0 \mathrm{e} 6$ seconds

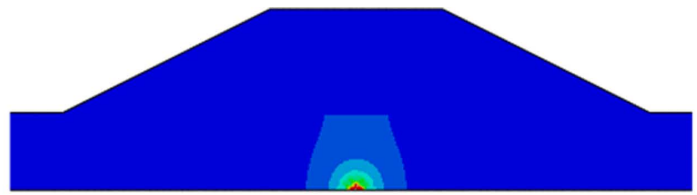

(b) $1.0 \mathrm{e} 6$ seconds

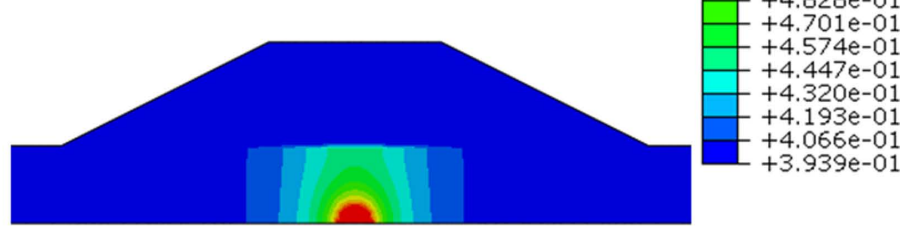

(d) $3.0 \mathrm{e} 6$ seconds

Figure 2. Spatial distribution of porosity with time.

The impact of internal erosion on the dike slope stability is evaluated by analyzing the evolution of the factor of safety $F_{s}$ by using the shear strength reduction method. Since $F_{s}$ is defined as the ratio between the actual soil shear strength and the minimum shear strength required to prevent failure, the simulations are run for a series of trial factors of safety $F^{\text {trial }}$ with $\phi$ adjusted according to the equation: 


$$
\phi^{\text {trail }}=\arctan \left(\frac{1}{F^{\text {trial }}} \tan \phi\right)
$$

Figure 3 shows the vertical displacement at point $\mathrm{E}$ obtained when the shear strength is progressively reduced in small steps, starting from a value of $F^{\text {trail }}=0.65$. It can be seen that with the amount of erosion increasing with time, the degradation of the soil develops, the factor of safety $F_{s}$ decreases, which indicates that the risk of mechanical instability increases.

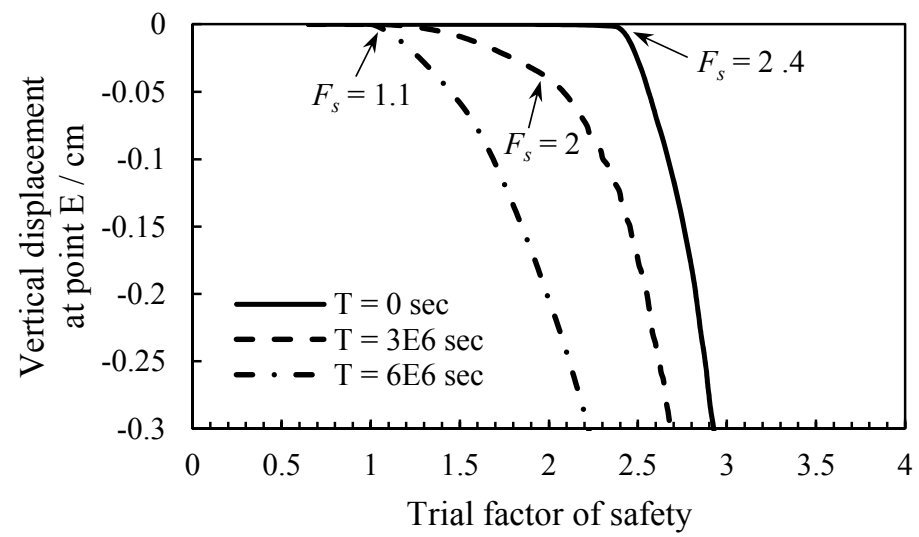

Figure 3. Vertical displacement as the trial factor of safety is increased for cases with different erosion time.

\section{CONCLUSION}

In this study, a numerical approach for modeling the internal erosion of soils is presented. The governing differential equations were formulated based on the mass balance of four constituents and then implemented into a finite element code. The internal erosion of the soil skeleton and the transport of fine particles in the fluid were modeled by the mass exchange between the solid skeleton and the pore water. Furthermore, a critical state based constitutive model was used to take into account the hydro-mechanical coupling effect induced by internal erosion. The example of a dyke was used to demonstrate the applicability of the model to simulate the evolution of internal erosion with time. The effects of internal erosion led to an increase of the soil porosity and to the degradation of the mechanical properties of the dyke foundation in time and space. The shear strength reduction method was adopted to evaluate the stability of the dike slope. The results show that the safety factor was reduced by the development of internal erosion, increasing the risk of mechanical instability. 


\section{REFERENCES}

Alboresha, R. (2016). "Evaluation of the impact of a cavity upon an earth dike (analytical and numerical approaches): Application to the Val d'Orléans area (France)." $\mathrm{PhD}$ thesis, Université de Lorraine.

Boer, R. (2000). "Contemporary progress in porous media theory." Applied Mechanics Reviews, 53(12), 323-370.

Cividini, A., and Gioda, G. (2004). "Finite-element approach to the erosion and transport of fine particles in granular soils." International Journal of Geomechanics, 4(3), 191-198.

Cividini, A., Bonomi, S., Vignati, G. C., and Gioda, G. (2009). "Seepage-induced erosion in granular soil and consequent settlements." International Journal of Geomechanics, 9(4), 187-194.

Fujisawa, K., Murakami, A., and Nishimura, S.-i. (2010). "Numerical analysis of the erosion and the transport of fine particles within soils leading to the piping phenomenon." Soils and foundations, 50(4), 471-482.

Reddi, L. N., Lee, I.-M., and Bonala, M. V. (2000). "Comparison of internal and surface erosion using flow pump tests on a sand-kaolinite mixture." Geotechnical Testing Journal, 23(1), 116-122.

Richart, F. E., Hall, J. R., and Woods, R. D. (1970). "Vibrations of soils and foundations."

Schrefler, B. (2002). "Mechanics and thermodynamics of saturated/unsaturated porous materials and quantitative solutions." Applied Mechanics Reviews, 55(4), 351-388.

Schaufler, A., Becker, C., and Steeb, H. (2013). "Infiltration processes in cohesionless soils." ZAMM-Journal of Applied Mathematics and Mechanics/Zeitschrift für Angewandte Mathematik und Mechanik, 93(2 3), 138-146.

Stavropoulou, M., Papanastasiou, P., and Vardoulakis, I. (1998). "Coupled wellbore erosion and stability analysis." International journal for numerical and analytical methods in geomechanics, 22(9), 749-769.

Sterpi, D. (2003). "Effects of the erosion and transport of fine particles due to seepage flow." international journal of Geomechanics, 3(1), 111-122.

Uzuoka, R., Ichiyama, T., Mori, T., and Kazama, M. "Hydro-mechanical analysis of internal erosion with mass exchange between solid and water." Proc., Proc. 6th International Conference on Scour and Erosion, Paris, France, 655-662.

Vardoulakis, I., Stavropoulou, M., and Papanastasiou, P. (1996). "Hydro-mechanical aspects of the sand production problem." Transport in porous media, 22(2), 225-244.

Wan, C. F., and Fell, R. (2004). "Investigation of rate of erosion of soils in embankment dams." Journal of geotechnical and geoenvironmental engineering, 130(4), 373-380.

Yin, Z.-Y., Xu, Q., and Hicher, P.-Y. (2013). "A simple critical-state-based double-yield-surface model for clay behavior under complex loading." Acta Geotechnica, 8(5), 509-523. 